\title{
Research on Safety Monitoring of Oxygen Cylinder Filling
}

\author{
Fangcheng WANG ${ }^{1,}$, , Lijing ZHANG ${ }^{1}$, Gang TAO ${ }^{1}$ \\ ${ }^{1}$ Jiangsu Key Laboratory of Hazardous Chemicals Safety and Control,College of safety science and Engineering,Nanjing Tech University \\ Nanjing 210009
}

\begin{abstract}
In recent years, the process of filling oxygen explosion accidents have occurred, causing great casualties and property losses. Great pressure on the safety production of enterprises, resulting in adverse effects on the society.This paper analyzes and summarizes the oxygen bottle filling process types and causes of the accident, found the shortcomings of traditional monitoring methods, and propose a new monitoring methodology, which is based on infrared detection technology oxygen cylinder filling process safety monitoring system. The method can accurately monitor the temperature changes of oxygen cylinder filling process, timely detection of anomalies in the filling process, provide some security for the oxygen cylinder filling operations..
\end{abstract}

\section{Introduction}

Oxygen as an ideal combustion gas is widely used in industrial production, with the rapid development of China's economy, the demand for oxygen is increasing, and oxygen-related accidents are increasing.At 15:50 on December 22, 2013, Shanxi Province, a company in cylinder explosion occurred, the place of the cylinder explosion is located in oxygen filling station, two people were killed and two people were injured[1]. For the Oxygen accident-prone situation, the party and the country attaches great importance.It has been issued many regulations, technical conditions and other documents about compression, gas production and cylinder filling areas[2].Enterprises and related researchers also carried out a detailed accident investigation and analysis, and gives many countermeasures in the aspect of management and technical.Based on previous research, this paper discusses the current lack of oxygen filling process management and monitoring technology, through practice and research to design a oxygen filling process safety monitoring system.

\section{2 infrared temperature measurement technology and principle}

\subsection{Infrared temperature measurement technology.}

Infrared temperature measurement technology was originally used in the military field, the current application in industrial production is also very extensive, especially in metallurgy, coal, electric power and other industries.Infrared temperature measurement technology has the following advantages:One is that its measurement does not interfere with the measured temperature field, which has a measurement with high accuracy; Second, the wide temperature range, no measurement limit in theory, can be measured relatively high temperatures; Third, the detector response time is short, fast response, easy to fast and dynamic measurement;Fourth, you do not have contact with the measured object, which is easy to operate; Fifth, tiny target temperature can be determined[3].

Currently, the device application of infrared temperature measurement technology is more, infrared thermometer,

${ }^{\mathrm{a} C}$ Corresponding author: 1006133376@qq.com 
infrared television, infrared camera and so on. Infrared thermometer is researched and developed at home and abroad earlier for its simple principle and structure,which is the most common form of non-contact type single-point thermometer. In practical application, its price is lower and the infrared thermometer for single point temperature measurement accuracy. It have been widely used in basic level for its simple operation and easy to master[4].

\subsection{Infrared temperature measurement principle.[5]}

In nature, an object that its temperature is above absolute zero $\left(-273^{\circ} \mathrm{C}\right)$, will radiate electromagnetic waves due to thermal motion of molecules, wherein the wavelength of $0.75 \mu \mathrm{m} \sim 1000 \mu \mathrm{m}$ band called infrared. At a given wavelength and temperature, radiation of the object having a maximum energy, which is called a blackbody, and its reflection coefficient is set to 1 , the other materials reflection coefficient is less than 1 , called gray body.

Blackbody spectral radiant power and temperature to meet the Planck radiation Theorem:

$$
p_{b}(\lambda T)=\frac{c_{1} \lambda^{-5}}{e^{\frac{c_{2}}{\lambda T}}-1}
$$

In Eq. $1,{ }_{b}(\lambda T)$ is the exit of the blackbody radiation, $\mathrm{W} /\left(\mathrm{m}^{2} \cdot \mu \mathrm{m}\right) ; \lambda$ is wavelength, $\mu \mathrm{m} ; \mathrm{T}$ is the absolute temperature, $\mathrm{K}$; $\mathrm{c} 1$, c 2 radiation constant.

From the Eq.1,we know that: (1) As the temperature increases, the stronger the radiation energy of the object; (2) at absolute temperature, the wavelength of the radiation emitted per unit area of a blackbody is blackbody radiation power.

Can be obtained the black-body radiation spectrum curve according to (2). From the graph, the radiation energy of the object is stronger with the temperature rising, the radiation peak shifts to shorter wavelength (to left), and meet the Wien displacement Theorem.

$$
T \cdot \lambda_{m}=2897.8 \mu m \cdot K
$$

Infrared temperature measurement must be based on wavelength division temperature range, high temperature in shortwave, low temperature in longwave, its sensitivity was high (steep curve), stronger anti-interference.

we get the Stephen - Boltzmann Theorem that blackbody radiation is proportional to the fourth power of the temperature,when the Planck theorem integral to wavelength from 0 to $\infty$.

$$
p_{b}(\lambda T)=\sigma T^{4}
$$

In Eq.3, $P_{b}(T)$ is the total blackbody radiation energy radiated from the blackbody per unit area and unit time at temperature $\mathrm{T}$, called the total irradiance, $\mathrm{W} / \mathrm{m}^{2} ; \quad \sigma$ is Stephen - Boltzmann constant; $T$ is the temperature of the object.

Blackbody radiation law is the theoretical basis of infrared temperature measurement technology. In general, gray body radiation power is always less than the blackbody radiation power that monochromatic radiation of the object is smaller than the total monochromatic radiant emittance of a blackbody. Their ratio $\varepsilon(\lambda)$ is called monochrome blackness of the object, namely the degree of actual objects close to the black body.

$$
\varepsilon(\lambda)=P(T) / P_{b}(\lambda T)
$$

Monochrome blackness of the object is a constant that not varies with wavelength. This object is called a gray body. The constant varies with the material. Only blackbody is 1 , and the general body of gray is between 0 and 1.So:

$$
\begin{gathered}
P(T)=\varepsilon P_{b}(\lambda T) \\
P(T)=\varepsilon \sigma T^{4}
\end{gathered}
$$

Then the measured target temperature is :

$$
T=\left(\frac{P(T)}{\varepsilon \sigma}\right)^{\frac{1}{4}}
$$

Eq.7 is the mathematical description of thermal radiation thermometry of an object. 


\section{Infrared temperature measurement system}

\subsection{System Design.}

Infrared temperature measurement system is based on infrared radiation characteristics of the object, using an infrared thermometer to receive infrared radiation $P(T)$ from oxygen cylinder surface. Get the electric signal corresponding to the cylinder surface temperature $\mathrm{T}$ with corresponding signal processing according to equation (7).
We obtained a corresponding digital signal of the temperature $\mathrm{T}$ by the $\mathrm{A} / \mathrm{D}$ conversion and then displayed on the LCD.Set an alarm temperature, when the temperature reaches the alarm, the alarm action alert. Notify the relevant personnel to evacuate or take emergency measures, safety interlock of filling system operation, automatic stop filling. System design block diagram shown in Figure 1.

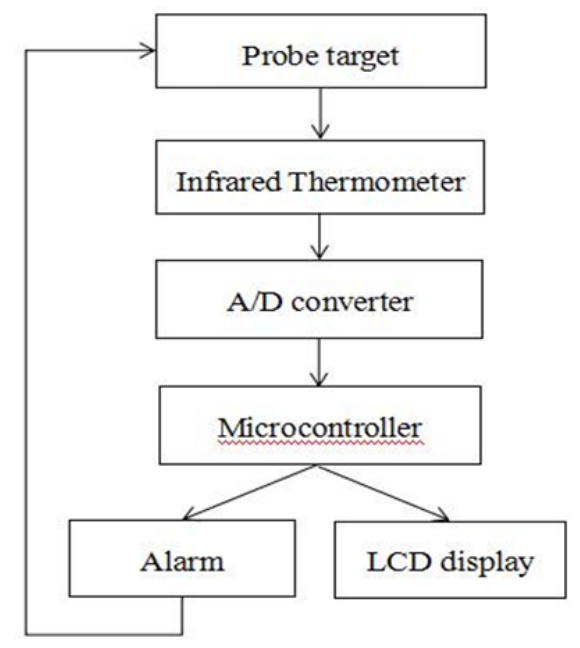

Figure 1 Block diagram of the overall infrared temperature measurement system design

\subsection{Each part of the system design.}

According to the total system design ideas, the following is the various parts of the system detailed design and presentation.

(1) infrared thermometer: Infrared thermometer consist of the optical system, photoelectric detectors, signal amplifiers and signal processors and other components.

The optical system receives goals infrared radiation in its field. Optical parts and its locations determine the size of the field of view. Infrared radiant energy focused on the optical detector and converted to electrical signals, amplified through the amplifier, and the signal would be corrected.

(2) A / D converter: The signal that Infrared thermometer get is an signal (analog signal), while the microcontroller can only handle digital signals. Therefore, the temperature of the electric signal first convert to digital signal by the A / D conversion,then the digital signal supplied to the microcontroller for data analysis and processing.

(3) Microcontroller : Digital signal got by the A / D converter is treated in the microcontrollert. SCM is an integrated circuit chip.It is a micro-computer system, which is small but perfect piece of silicon, consist of the CPU has a data processing capability, random access memory RAM, a read only memory ROM, a variety of $\mathrm{I} / \mathrm{O}$ port and interrupt system, timers/counters, etc. It complete the process by the use of VLSI technology[6]. MCU of this system needs to be done the following functions: First, accurate measured target temperature, and sent to LCD display; Second, can set the temperature by ourselves, send alarm signal to alarm when the real temperature is over; Third, can set a alarm temperature rise speed, when the actual temperature rises too fast, alarm alarm; Fourth,fuction of data storage; Fifth, input and output functions.You can print and copy a variety of data inside memory. 
(4) LCD display: Liquid crystal display (LCD) displays real-time monitoring of temperature. Suitable display size and suitable resolution to meet the basic requirements of a clear and accurate display.

(5) Alarm: Alarm receives the alarm signal from the microcontroller and alerts. Audible alarm is one of the alarm to alert personnel via visual and auditory stimuli double.

\section{Example applications}

In order to verify the feasibility of infrared temperature measurement system,I choose a oxygen cylinder filling Enterprise as a pilot. The company has an oxygen filling line.

\subsection{Selection of major equipment}

(1) infrared thermometer

Parameters of the infrared thermometer selected are shown in Table 1.

(2) Display control device

Parameters of display control instrument selected in are shown Table 2.

Table 1 The main parameters of infrared thermometer

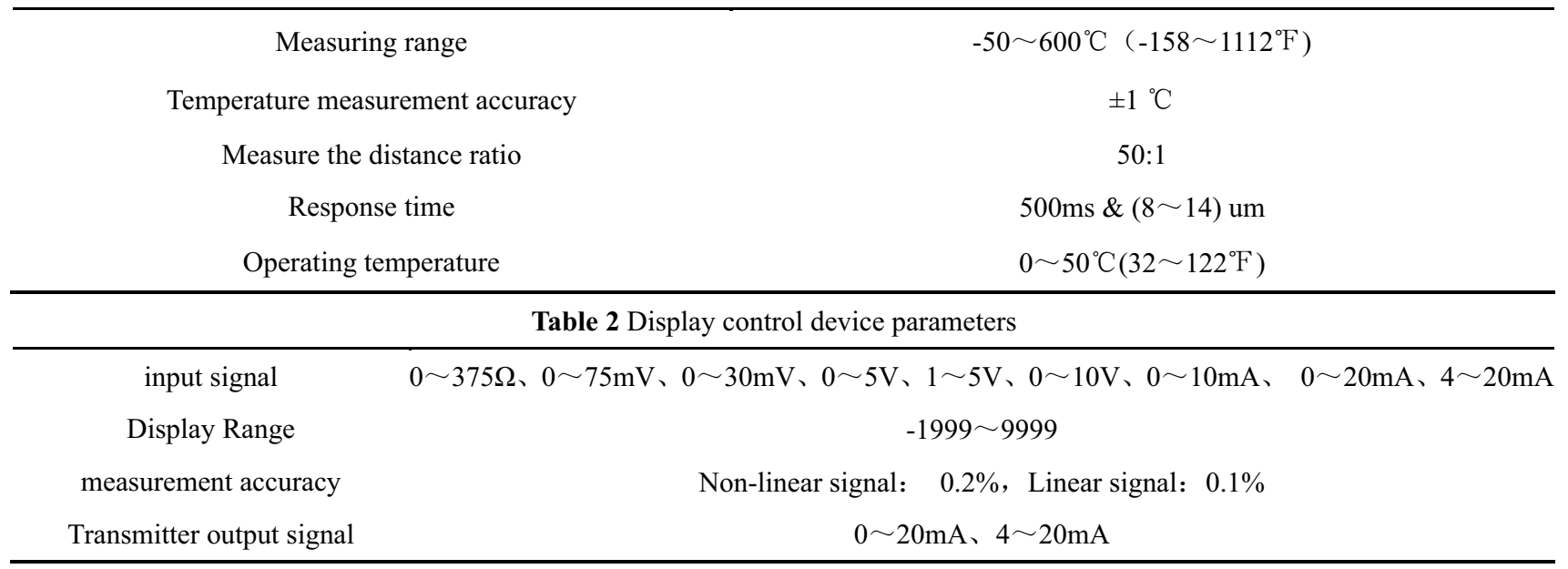

\subsection{Molding equipment assembly.}

According to the system design, the assembled device is

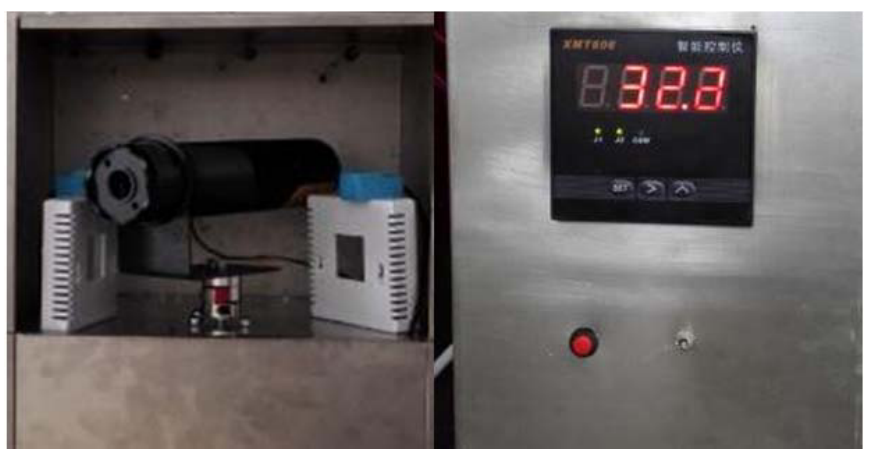

Figure 2 Front and side view of the device

\subsection{System operation.}

After the system is assembled, one oxygen cylinder for being in the process of filling is in for real-time monitoring by the system. The system runs smoothly and normal. Part of the monitoring data is as follows:

(1)Alarm temperature is set to 60 centigrade, room temperature is 20 centigrade. See Table 3.
(2)Alarm temperature is set to 45 centigrade, room temperature is 20 centigrade. See Table 4.

(3)Alarm temperature is set to 30 centigrade, room temperature is 20 centigrade. See Table 5.

The above analysis of the data shows that: First, With the filling pressure increases, the surface temperature of the oxygen cylinder increases;Second, Pre-filled cylinder temperature rises faster, the late temperature rise 
slowly; Third, Normal filling, oxygen cylinder surface temperature will not exceed 60 centigrade; Fourth, the average temperature of the three measurements was $325.4 \mathrm{~K}$ ( 52.4 centigrade) in the maximum filling pressure.

Table 3 The alarm monitoring data of the temperature of 60 centigrade

\begin{tabular}{cccccccc}
\hline Filling Pressure[P/MPa] & 3 & 5 & 7 & 9 & 11 & 13 & 14.7 \\
\hline Temperature display[T/K] & 294.8 & 309.3 & 316.1 & 319.9 & 322.5 & 324.3 & 325.4 \\
Alarm [Yes / No] & No & No & No & No & No & No & No \\
\hline
\end{tabular}

Table 4 The alarm monitoring data of the temperature of 45 centigrade

\begin{tabular}{cccccccc}
\hline Filling Pressure[P/MPa] & 3 & 5 & 7 & 9 & 11 & 13 & 14.7 \\
\hline Temperature display[T/K] & 295.1 & 309.5 & 316.0 & 320.1 & 322.4 & 324.6 & 325.2 \\
Alarm [Yes / No] & No & No & No & Yes & Yes & Yes & Yes \\
\hline
\end{tabular}

Table 5 The alarm monitoring data of the temperature of 30 centigrade

\begin{tabular}{cccccccc}
\hline Filling Pressure $[P / \mathrm{MPa}]$ & 3 & 5 & 7 & 9 & 11 & 13 & 14.7 \\
\hline Temperature display[T/K] & 295.3 & 309.7 & 316.3 & 319.8 & 322.2 & 324.4 & 325.5 \\
Alarm [Yes / No] & No & Yes & Yes & Yes & Yes & Yes & Yes \\
\hline
\end{tabular}

\section{Conclusion}

From the oxygen cylinder explosion, we analyze the cause and type of the explosion and pointed out shortcomings of current oxygen cylinder filling process safety monitoring. Designed a oxygen cylinder filling process monitoring system based on infrared detection technology. Its practical and scientific was verified by examples. The results show that the use of infrared temperature measurement system for oxygen cylinder filling process temperature monitoring is scientific and feasible. The system can accurately detect and display the oxygen cylinder filling process temperature, and alarm when the temperature exceeds the set alarm. Can provide some security to the oxygen cylinder filling process.It have greater economic benefits to the enterprise production safety.

\section{References}

1. Yang $\mathrm{Xu}$. Reason analysis of oxygen cylinder explosion accident. China Special Equipment Safety, 30(009) (2014) 64-68

2. Ye yi. On safety issues of filling oxygen cylinders. Cryogenic technology.6(2005)46-48

3. Li Jun, Liu Meidong, Zeng Yike.Research on Non-contact infrared temperature measurement.PIEZOELECTRICS

$\&$ ACOUSTOOPTICS.23(3) (2001) 202-205

4. Qian Wei.Design of real-time on-line monitoring system for switchgear temperature based on infrared sensor.Nanjing University of Science Master Thesis.(2009)

5. Yan Ming,Peng Chiwu,Yan Yonghong.Principle and Error Analysis of Infra-Red Temperature Measurement.Journal of Hunan University(Natural Sciences).31(5) (2004) 110-112

6. Zhang Xuezhong,Wang Xiujun.Closed-loop control of stepper motor based microcontroller At89S52.AGRICULTURAL EQUIPMENT \& TECHNOLOGY. 3(2011) 31-32. 